\title{
GPS radio sources: new optical observations and an updated master list ${ }^{\star}$
}

\author{
A. Labiano ${ }^{1,2}$, P. D. Barthel ${ }^{1}$, C. P. O'Dea ${ }^{3}$, W. H. de Vries ${ }^{4,5}$, I. Pérez ${ }^{1}$, and S. A. Baum ${ }^{6}$ \\ 1 Kapteyn Astronomical Institute, Groningen, 9700 AV, The Netherlands \\ e-mail: labiano@damir.iem.csic.es \\ 2 Departamento de Astrofísica Molecular e Infrarroja, Instituto de Estructura de la Materia (CSIC), Madrid, Spain \\ 3 Department of Physics, Rochester Institute of Technology, Rochester, NY 14623, USA \\ ${ }^{4}$ University of California, Davis, CA 95616, USA \\ 5 Lawrence Livermore National Laboratory / IGPP, Livermore, CA 94550, USA \\ ${ }^{6}$ Center for Imaging Science, Rochester Institute of Technology, Rochester, NY 14623, USA
}

Received 4 August 2006 / Accepted 9 November 2006

ABSTRACT

\begin{abstract}
Aims. We identify optical counterparts, address uncertain identifications and measure previously unknown redshifts of the host galaxies of candidate GPS radio sources, and study their stellar populations.

Methods. Long slit spectroscopy and deep optical imaging in the $B, V$ and $R$ bands, obtained with the Very Large Telescope.

Results. We obtain new redshifts for B0316+161, B0407-658, B0904+039, B1433-040, and identify the optical counterparts of B0008-421 and B0742+103. We confirm the previous identification for B0316+161, B0407-658, B0554-026, and B0904+039, and find that the previous identification for B0914+114 is incorrect. Using updated published radio spectral information, we classify as non GPS the following sources: B0407-658, B0437-454, B1648+015. The optical colors of typical GPS sources are consistent with single instantaneous burst stellar population models but do not yield useful information on age or metallicity. A new master list of GPS sources is presented.
\end{abstract}

Key words. galaxies: active - galaxies: distances and redshifts - galaxies: quasars: emission lines - galaxies: general

\section{Introduction}

GigaHertz Peaked Spectrum (GPS) radio sources are fairly common - O'Dea (1998) lists a 10\% fraction in high frequency selected catalogs. In the current paradigm, the extended radio galaxies and quasars are unified with the compact, coredominated quasars and BL Lac-objects through the combined effects of radio jet orientation and anisotropic obscuration (e.g., Urry \& Padovani 1995). These objects are considered to be mature, well developed radio sources. It is likely that GPS objects are young radio sources that will evolve into the $10-100 \mathrm{kpc}$ scale objects. Studies of radio galaxy evolution suggest that the GPS sources can evolve into the larger scale radio galaxies, dimming in radio luminosity as they expand (e.g., Fanti et al. 1995; Begelman 1996; Readhead et al. 1996; De Young 1997; Kaiser et al. 1997; Kaiser \& Alexander 1997; O’Dea \& Baum 1997; Snellen et al. 2000). Multi-color optical as well as near-IR imaging (e.g., O'Dea et al. 1996; Snellen et al. 1996; de Vries et al. 2000a) have shown that host galaxy colors of nearby GPS objects are indeed consistent with non- or passively evolving ellipticals, with absolute magnitudes comparable to the brightest cluster members, similar to the hosts of intermediate sized and large radio source classes. Determination of the rest-frame broad-band colors (which requires redshifts) in connection with stellar population synthesis modeling has proven essential for these investigations (e.g., de Vries et al. 2000b).

O'Dea et al. (1991) presented a list of candidate GPS radio sources. About half of those sources had unknown redshifts

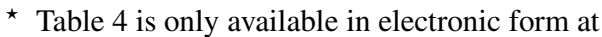
http: //www . aanda.org and several lacked an optical counterpart. The sample has been updated with new identifications and redshifts since then (e.g., de Vries et al. 1997). However, the optically faint part of the sample requires the use of $8 \mathrm{~m}$ class telescopes. Our goal in this paper is to increase the number of optical identifications, measure new redshifts and remove non GPS sources from the current samples, using updated radio data from the literature. Using the ESO VLT, we have therefore observed 8 unidentified and 5 tentatively identified objects from the master list of O'Dea et al. (1991).

We here present these observations of sources with unknown or uncertain redshifts or optical counterparts, and increase the number of confirmed GPS sources (up to 74, out of 95 candidates) of the complete O'Dea et al. (1991) list. We give new host identifications (down to $R \sim 25$ ) and obtain several new redshifts. Using these, as well as literature research and our earlier data, we update the O'Dea et al. (1991) master list. This updated master list is presented in a concluding table. We use $H_{0}=71, \Omega_{\mathrm{M}}=0.27, \Omega_{\Lambda}=0.73$ (Spergel et al. 2003) throughout the paper.

\section{Observations and data reduction}

The sample was observed during two nights (January 30 to 31,2000 , and December 16 to 17, 2001) using VLT's FORS1/UT1 and FORS2/UT4 (Appenzeller et al. 1998). We obtained long slit spectroscopy using grism 150I with order separators OG590 and GG375, obtaining a $230 \AA / \mathrm{mm}$ dispersion (5.52 $\AA /$ pixel) and covering wavelengths 6000-11000 (OG590) and 3850-7500 $\AA$ (GG375). For the imaging we 
Table 1. Source list and exposure times (in seconds).

\begin{tabular}{lcccc}
\hline \hline Name & Spectroscopy & $B$ & $V$ & $R$ \\
\hline B0008-421 & 1800 & - & - & 600 \\
B0316+161 & 3000 & 300 & 600 & - \\
B0407-658 & 1500 & 600 & 300 & 300 \\
B0437-454 & 1800 & - & - & - \\
B0554-026 & - & 300 & 300 & 300 \\
B0742+103 & 3000 & - & - & 600 \\
B0904+039 & 3000 & 600 & 300 & - \\
B0914+114 & $1200^{a}$ & 600 & 480 & - \\
B1045+019 & 1800 & - & - & - \\
PMN J1300-1059 & 900 & - & - & - \\
B1433-040 & 1200 & - & - & - \\
B1601-222 & 1200 & - & - & - \\
B1648+015 & 1350 & - & - & - \\
\hline
\end{tabular}

${ }^{a}$ Unrelated galaxy. See notes on individual source for details. ${ }^{b}$ PMN J1300-1059 was chosen from the NVSS and WISH surveys (Condon et al. 1998; De Breuck et al. 2002).

used a $R$-Johnson-Cousins filter for the January 2000 run. For the December run, we used the $B, V$ - Johnson-Cousins and $R$-Special filters ${ }^{1}$. We covered a 6.8 arcmin field with seeing ranging from $0.5^{\prime \prime}$ to $0.7^{\prime \prime}$ for the January run and $0.5^{\prime \prime}$ to $1.1^{\prime \prime}$ for the December run. The pixel scale of FORS is $0.25^{\prime \prime} /$ pixel. Table 1 lists the exposure times.

Standard data reduction was performed using IRAF routines. All the spectra were corrected for bias, flat-field and sky subtracted. Wavelength calibration was done using internal arc lamps. The flux calibration and removal of atmospheric lines were performed using the spectrophotometric standards GD50 and GD108.

The calibration of the imaging data was different for the January and December runs. Both runs were corrected for bias and flat-field. The January observations were done during a photometric night. We took flat field images and observed the Landolt (1992) standard fields PG1323-086 and SA 95. No useful standard fields or flat fields were observed for the - non photometric - December run. To correct from flat-field these images, we averaged all the observations in each filter separately to create artificial flat-field images, which we used for the correction. As standard stars, we chose unsaturated field stars with data in the Second Guide Star Catalogue (GSC2.2, McLean et al. 1998), about 40 stars in total. The GSC stars have available magnitudes in the photographic $F$ and $J$ bands. The transformation to the Johnson-Cousins filters was performed following Kent (1985). The January images were taken only in $R$-band. For the December run, most of the standards lacked color information to perform (first order) color coefficient corrections to our apparent magnitudes. We could only fit the zero point magnitudes in each band. The errors on the zero point are: 0.042 for the January observations ( $R$-band) and $0.38,0.17,0.10$ for the December observations ( $R, B, V$-bands respectively).

\section{Results}

\subsection{Identifications}

The astrometry was performed using the GSC2.2 catalog as reference. It was possible to make accurate (usually with $1 \sigma$ error $<0.5$ arcsec) positional determinations for the candidate optical counterparts. The radio positions are from the

1 The $R$-Special filter on FORS2 is a Johnson-Cousins filter with a slightly shortened red end to avoid sky emission lines.
VLA calibrator manual (B0008-421, B0316+161, B0742+103) and NED (B0407-658, B0554-026, B0904+039, B0914+114). As in de Vries et al. (1995, 2000b), we use the likelihood ratio defined by de Ruiter et al. (1977):

$R_{1}=\sqrt{\frac{\Delta \alpha^{2}}{\sigma_{\alpha}^{2}}+\frac{\Delta \delta^{2}}{\sigma_{\delta}^{2}}}$

where $\Delta \alpha$ and $\Delta \delta$ are the measured offsets in RA and Dec between the optical and radio positions, $\sigma_{\alpha}^{2}$ and $\sigma_{\delta}^{2}$ are the sums of the squared $1 \sigma$ errors in the optical and radio positions. The probability that a true optical counterpart has an $R_{1}$ value larger than some $R_{0}$ is given by $P\left(R_{1}>R_{0}\right)=\mathrm{e}^{-0.5 R_{0}^{2}}$. An $R_{1}$ value less than three indicates a probability of less than $1 \%$ of a false identification (assuming that the optical counterpart is the object closest to the radio position). Most of our identifications have $R_{1}$ values smaller than 3 and are likely to be correct. The optical position used in the calculation of $R_{1}$, and listed in Table 2, corresponds to the center of the source, fitted with IRAF task Ellipse. The identification results are listed in Table 2 and finding charts for the sources are presented in Fig. 4.

\subsection{Magnitudes}

The magnitudes were extracted performing aperture photometry. We used apertures large enough to include all light from the object (typically a diameter of $\sim 10$ pixels) but minimizing the contribution from sky. The magnitudes are corrected for Galactic extinction, following Schlegel et al. (1998).

B0008-421 and B0742+103 were observed in the $R$-Johnson-Cousins filter in the January 2000 - photometric - night. The photon noise increases the error in magnitude to 0.3 and 0.1 for B0008-421 (with lower signal to noise) and B0742+103 respectively (Table 2).

The rest of the images were taken in the - non photometric - December 2001 run. As judged from internal consistency of field stars with known magnitudes, our photometric accuracy varies between 0.1 and $0.4 \mathrm{mag}$. The main source of error for this run comes from the uncertainties in the magnitudes of the stars used in the calibration: $0.17,0.10$ and 0.38 for $B, V$ and $R$ bands respectively. A conservative $(5 \sigma)$ detection limit is magnitude $\sim 25.5$ for $R, V$-bands and $\sim 26$ for $B$-band, for all sources.

Comparison with the Hubble diagram (O'Dea et al. 1996; Snellen et al. 2002; and Fig. 1) show that the $R$-magnitudes we measure are consistent with previous observations of GPS hosts. The $V$-magnitudes of the new optical counterparts are consistent with those found by di Serego-Alighieri et al. (1994) for hosts of radio sources, although our sources tend to be slightly fainter. We have not found published $B$ magnitudes for comparison.

\subsection{Spectra and redshifts}

We took spectra of 16 sources to measure their redshifts. Table 3 and Fig. 3 show those spectra where emission features were found. We measure redshifts for B0316+161, B0407-658, B0904+039, B1433-040 (with a conservative error of \pm 0.001 ) and (the incorrect ID for) B0914+114. Some sources show possible emission lines but their redshifts are uncertain (see notes on each individual object below): B0008-421, PMN J1300-1059, and B1045+019. The spectra were too noisy to find emission features for B0437-454, B0742+103, B1601-222 and B1648+015. 
Table 2. Positions of the optical counterparts.

\begin{tabular}{ccccccl}
\hline \hline & \multicolumn{2}{c}{ Radio (J2000) } & \multicolumn{2}{c}{ Optical (J2000) } & & \\
\cline { 2 - 5 } Source & RA & Dec & RA & Dec & $R_{1}$ & Magnitude \\
\hline B0008-421 & $00: 10: 52.52$ & $-41: 53: 10.8$ & $00: 10: 52.53$ & $-41: 53: 10.6$ & 2.1 & $R 24.3 \pm 0.3 \pm 0.04$ \\
B0316+161 & $03: 18: 57.80$ & $+16: 28: 32.7$ & $03: 18: 57.82$ & $+16: 28: 32.9$ & 2.0 & $B 23.0 \pm 0.1 \pm 0.2$ \\
B0316+161 & $"$ & $"$ & $03: 18: 57.82$ & $+16: 28: 32.6$ & 2.0 & $V 23.4 \pm 0.1 \pm 0.1$ \\
B0407-658 & $04: 08: 20.38$ & $-65: 45: 09.1$ & $04: 08: 20.37$ & $-65: 45: 09.0$ & 1.3 & $B 22.5 \pm 0.04 \pm 0.2$ \\
B0407-658 & $"$ & $"$ & $04: 08: 20.41$ & $-65: 45: 08.6$ & 3.4 & $V 21.4 \pm 0.03 \pm 0.1$ \\
B0407-658 & $"$ & $"$ & $04: 08: 20.41$ & $-65: 45: 09.4$ & 3.4 & $R 20.2 \pm 0.06 \pm 0.4$ \\
B0554-026 & $05: 56: 52.62$ & $-02: 41: 05.5$ & $05: 56: 52.61$ & $-02: 41: 05.3$ & 2.6 & $B 18.3 \pm 0.01 \pm 0.2$ \\
B0554-026 & $"$ & $"$ & $05: 56: 52.59$ & $-02: 41: 05.5$ & 3.3 & $V 17.5 \pm 0.02 \pm 0.1$ \\
B0554-026 & $"$ & $"$ & $05: 56: 52.62$ & $-02: 41: 05.5$ & 3.2 & $R 16.4 \pm 0.01 \pm 0.4$ \\
B0742+103 & $07: 45: 33.06$ & $+10: 11: 12.7$ & $07: 45: 33.06$ & $+10: 11: 12.5$ & 1.9 & $B 24.0 \pm 0.1 \pm 0.2$ \\
B0742+103 & $"$ & $"$ & $07: 45: 33.06$ & $+10: 11: 12.3$ & 1.9 & $V 23.8 \pm 0.2 \pm 0.1$ \\
B0742+103 & $"$ & $"$ & $07: 45: 32.97$ & $+10: 11: 12.8$ & 3.9 & $R 23.1 \pm 0.1 \pm 0.04$ \\
B0904+039 & $09: 06: 41.05$ & $+03: 42: 41.5$ & $09: 06: 41.03$ & $+03: 42: 42.0$ & 3.3 & $V 24.9 \pm 0.3 \pm 0.1$ \\
B0914+114 & $09: 17: 16.39$ & $+11: 13: 36.5$ & $09: 17: 16.53$ & $+11: 13: 31.4$ & 52.6 & $B 21.3 \pm 0.04 \pm 0.2$ \\
B0914+114 & $"$ & $"$ & $09: 17: 16.54$ & $+11: 13: 32.3$ & 44.4 & $V 19.9 \pm 0.01 \pm 0.1$ \\
\hline
\end{tabular}

The radio positions are from the VLA calibrator manual (B0008-421, B0316+161, B0742+103) and NED (B0407-658, B0554-026, B0904+039, B0914+114). The typical uncertainties in the radio positions are $0.01^{\prime \prime}$ in Declination and $0.001 \mathrm{~s}$ in Right Ascension. The $R_{1}$ factor is the likelihood ratio (see text for details). The optical coordinates of B0914+114 correspond to the galaxy previously - and incorrectly - identified as the counterpart (see notes on individual source). The errors in the magnitudes are divided in photon noise (first error) and calibration (second error). The magnitudes are corrected for Galactic extinction, following Schlegel et al. (1998). B0316+161 magnitudes may be affected by close-by $\left(\sim 3^{\prime \prime}\right)$ objects.

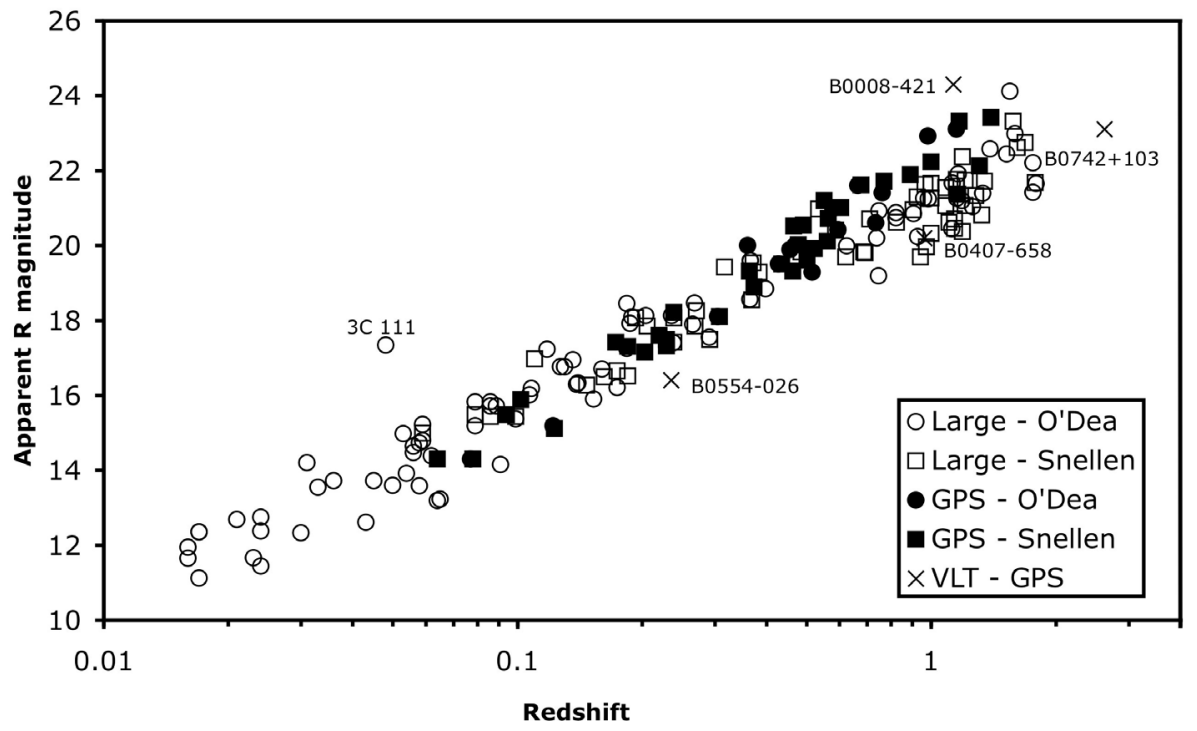

Fig. 1. $R$-band Hubble diagram for GPS and large radio galaxies. Data from O'Dea et al. (1996), Snellen et al. (2002) and our VLT measurements (marked with X). Redshifts of B0742+103 and B0554-026 from Best et al. (2003) and de Vries et al. (2000b). 3C 111 is obscured by the Galactic dark cloud complex Taurus B (e.g., Sguera et al. 2005, and references therein).

\subsection{Stellar population synthesis models}

GPS radio sources are found to live in passively evolving elliptical galaxies, and have colors consistent with those predicted by stellar synthesis models (e.g., O'Dea et al. 1996; de Vries et al. 1998; Snellen et al. 2002). Most of our objects are identified with galaxies and not quasars. Therefore, most of the optical radiation can be associated with stars in the host.

We have compared our redshift and magnitude measurements with the Bruzual \& Charlot (2003) stellar population synthesis models. The models have been run using the Chabrier (Chabrier 2003) initial mass function, metallicities $Z=0.008$, $Z=0.02, Z=0.05$ and ages (time since the initial starburst) ranging from 5 to 12.5 Gyr. Different star formation histories have been used: instantaneous burst, exponentially declining, and constant star formation. We fitted colors $B-V$ and $V-R$ for the sources where we had color information.
Constant and exponentially declining star formation models seem to be ruled out by our data. The data points and best models disagree by roughly 0.5 to $1 \mathrm{mag}$.

The optical color data are in better agreement with the passively evolving instantaneous burst models (Fig. 2), consistent with previous works (e.g., de Vries et al. 2000a). Our data seem to favor models with ages of $\sim 12$ Gyr and metallicity 0.008 . However, the known degeneracy between metallicities and ages suggests that these results should be viewed with caution. In addition, we note that our HST UV observations suggest that many GPS hosts contain a small contribution from recent star formation (Labiano et al. 2006, 2007, in prep.).

\section{Notes on individual sources}

B0008-421: following unsuccessful attempts by di Serego-Alighieri et al. (1994), de Vries et al. (1995) 
Table 3. Line identifications and redshifts.

\begin{tabular}{|c|c|c|c|c|c|c|c|c|}
\hline Name & ID & Line & Wavelength $(\AA)$ & Redshift & Mean & Flux & $F W H M$ & Log Power $5 \mathrm{GHz}$ \\
\hline \multirow[t]{2}{*}{ B0008-421 } & $\mathrm{G}$ & {$[\mathrm{Ne} \mathrm{V}] 3425 ?$} & 7294 & 1.130 & $1.130^{*}$ & 0.3 & 29 & 26.7 \\
\hline & & [O II $] 3727 ?$ & 7940 & 1.130 & & 0.7 & 39 & \\
\hline \multirow[t]{5}{*}{ B0316+161 } & $\mathrm{G}$ & [O II] 3727 & 7102 & 0.906 & 0.907 & 5.4 & 21 & 27.6 \\
\hline & & [Ne III] 3869 & 7381 & 0.908 & & 1.8 & 32 & \\
\hline & & $\mathrm{H}_{\beta} 4861$ & 9277 & 0.908 & & 4.7 & 49 & \\
\hline & & [O III] 4959 & 9464 & 0.908 & & 8.3 & 35 & \\
\hline & & [O III] 5007 & 9544 & 0.906 & & 33 & 51 & \\
\hline \multirow[t]{8}{*}{ B0407-658 } & $\mathrm{G}$ & {$[\mathrm{Ne} \mathrm{V}] 3425$} & 6721 & 0.962 & 0.962 & 11 & 25 & 27.7 \\
\hline & & [O II $] 3727$ & 7314 & 0.962 & & 6.9 & 29 & \\
\hline & & [Ne III] 3869 & 7595 & 0.963 & & 2.0 & 44 & \\
\hline & & [Ne III] 3967 & 7787 & 0.963 & & 0.7 & 29 & \\
\hline & & $\mathrm{H}_{\gamma} 4102$ & 8048 & 0.962 & & 0.3 & 18 & \\
\hline & & $\mathrm{H}_{\beta} 4861$ & 9538 & 0.962 & & 4.4 & 52 & \\
\hline & & [O III] 4959 & 9731 & 0.962 & & 43 & 51 & \\
\hline & & [O III] 5007 & 9823 & 0.962 & & 16 & 54 & \\
\hline \multirow[t]{3}{*}{ B0904+039 } & $\mathrm{G}$ & [O II $] 3727$ & 6822 & 0.830 & 0.830 & 1.9 & 17 & 24.9 \\
\hline & & [O III] 4959 & 9078 & 0.831 & & 1.8 & 26 & \\
\hline & & [O III] 5007 & 9162 & 0.830 & & 4.4 & 26 & \\
\hline \multirow[t]{6}{*}{ B0914+114 } & $\mathrm{G}$ & Break 4000 & 4711 & 0.178 & 0.178 & - & - & - \\
\hline & & $\mathrm{H} \beta 4861$ & $5690 ?$ & $0.171 ?$ & \pm 0.005 & - & - & \\
\hline & & $\operatorname{Mg} b 5200$ & 6115 & 0.183 & & - & - & \\
\hline & & [N II] 6548 & 7667 & 0.171 & & - & - & \\
\hline & & $\mathrm{H} \alpha 6563$ & 7772 & 0.184 & & - & - & \\
\hline & & [S II] 6716 & 7952 & 0.184 & & - & - & \\
\hline \multirow[t]{3}{*}{ B1045+019 } & & [O II $] 3727$ & 6297 & 0.689 & $0.689^{*}$ & 0.4 & 12 & 26.6 \\
\hline & & $\mathrm{H}_{\gamma} 4102$ & 7327 & 0.688 & & 0.3 & 12 & \\
\hline & & [He II] 4686 & 7918 & 0.689 & & 0.3 & 29 & \\
\hline PMN J1300-1059 & $\bar{Q} ?$ & Mg II 2799 & 6385 & 1.283 & $1.283^{*}$ & 10 & 125 & - \\
\hline \multirow[t]{8}{*}{ B1433-040 } & $\mathrm{Q}$ & [O II $] 3727$ & 6694 & 0.796 & 0.795 & 17 & 19 & 26.3 \\
\hline & & [Ne III] 3869 & 6949 & 0.796 & & 32 & 54 & \\
\hline & & [Ne III] 3967 & 7122 & 0.795 & & 10 & 21 & \\
\hline & & $\mathrm{H}_{\delta} 4102$ & 7355 & 0.794 & & 23 & 84 & \\
\hline & & $\mathrm{H}_{\gamma} 4341$ & 7790 & 0.795 & & 76 & 113 & \\
\hline & & $\mathrm{H}_{\beta} 4861$ & 8724 & 0.795 & & 347 & 192 & \\
\hline & & [O III] 4959 & 8900 & 0.795 & & 63 & 30 & \\
\hline & & [O III] 5007 & 8987 & 0.795 & & 121 & 27 & \\
\hline
\end{tabular}

For B1433-040, the redshift is measured with the narrow emission profiles. The * means the redshift estimation is uncertain for that source, see text for details. The $F W H M$ listed is the observed $F W H M$ corrected for the $F W H M$ of the instrumental spectrum. Fluxes in $10^{-16} \mathrm{erg} / \mathrm{cm}^{2} / \mathrm{s}$. A conservative error for $z$ is \pm 0.001 . The B0914+114 results correspond to the unrelated galaxy. It shows emission and absorption lines mixed so the centers are more uncertain than in the other sources (the $1 \sigma$ error in $z$ is listed below the mean value) and line properties will depend on the stellar population model. The last column lists the radio power in $\mathrm{W} / \mathrm{Hz}$ of the source for the redshifts listed. The $5 \mathrm{GHz}$ fluxes are from O'Dea (1998) and Wright \& Otrupcek (1990).

and Costa (2001), we have now identified the bright radio source B0008-421 with an $R=24.3$, somewhat diffuse galaxy (Fig. 4). This magnitude is consistent with previous nondetections (which had detection limits down to magnitude 23). We detect a faint object, $0.2^{\prime \prime}$ from the VLBI position. The spectrum is consequently faint and very noisy making it difficult to distinguish emission lines from noise (Fig. 3). Comparison with the GPS Hubble diagram (O'Dea et al. 1996, see Fig. 1) shows that for an apparent $R$ magnitude $\sim 24$, the expected redshift is between 1 and 2 . We find two dubious emission lines consistent with this range of redshift: $[\mathrm{Ne} \mathrm{V}] \lambda 3425$ and [O II] $\lambda 3727$ at $7294 \AA$ and $7940 \AA$, which would correspond to a redshift of $z=1.130$.

B0316+161: our deep image (Fig. 4) confirms the earlier identification of this well known GPS radio source (also known as CTA21), by Stanghellini et al. (1993). We find a rather compact host, and note that the object seen $8^{\prime \prime} \mathrm{NNW}$ of the CTA21 identification in the Stanghellini et al. image is unrelated. The relative faintness of B0316 +161 , and the proximity of bright objects, may be affecting our measured magnitudes. The spectrum shows a weak continuum with bright [O II] and [O III] lines (Fig. 4). We measure $z=0.907$ based on five lines.

B0407-658: Stickel et al. (1996) identified the optical counterpart of this radio source as a galaxy. The spectrum (Fig. 3) shows a faint continuum spectrum where we identify nine emission lines at $z=0.962$. The emission line gas is more extended $\left(3.2^{\prime \prime}, 25 \mathrm{kpc}\right)$ along the slit than the stellar continuum. We observe no shift in wavelength along the spatial direction in the spectra. Our resolution $(\sim 18 \AA)$ yields a velocity resolution limit of $\sim 500 \mathrm{~km} \mathrm{~s}^{-1}$ for the central part of the spectrum. Recent radio spectral data (NED) ${ }^{2}$ show no peak around $1 \mathrm{GHz}$ for this source, suggesting a CSS or larger radio source. However, the source is unresolved in ATCA observations (resolution $\sim 5 \times 3^{\prime \prime}$ Morganti et al. 1993).

B0437-454: the spectrum shows a bright continuum but no emission or absorption lines. Updated radio data indicate that the radio spectrum of B0437-454 is only marginally peaked. In addition, pronounced variability was reported: $0.6 \mathrm{Jy}$ vs. $1.4 \mathrm{Jy}$

\footnotetext{
${ }^{2}$ NASA/IPAC Extragalactic Database.
} 

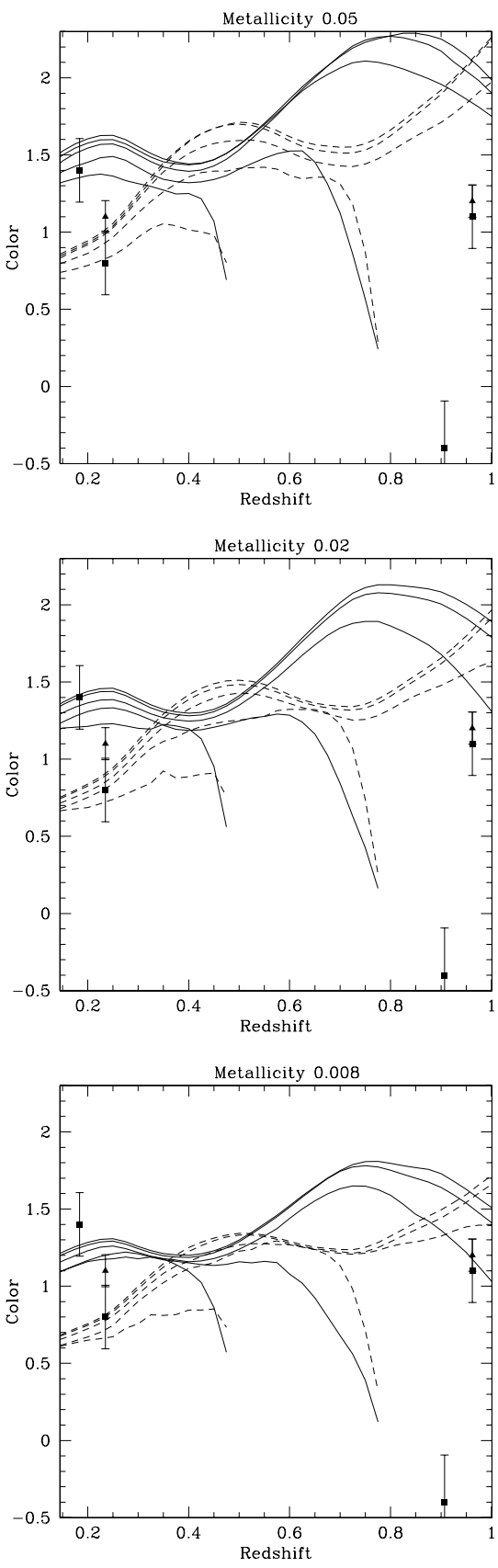

Fig. 2. Instantaneous, single burst stellar population synthesis models (lines) and observational colors (points) for our data. Squares and solid lines represent $B-V$, triangles and dashed lines represent $V-R$. There are five ages represented for each color: 5.0, 6.8, 9.3, 11.5 and $12.5 \mathrm{Gyr}$. The age of the population increases from bottom to top. The discrepant data point corresponds to B0316+161 (at $z=0.907$ ). However, the measured color of B0316+161 may be affected by bright nearby objects.

at $5 \mathrm{GHz}$ (e.g., Wright et al. 1994; Wright \& Otrupcek 1990). Also, given the identification with an optical point source, we conclude that the object is probably a BL Lac object and should be removed from the GPS list.

B0554-026: we confirm the identification of de Vries et al. (2000b) $(z=0.283)$ of this galaxy. We find a rather bright $(B=$ $18.3, V=17.5)$ and extended source $\left(\sim 5^{\prime \prime}\right)$. We obtain $R=$ 16.4 but this band may be affected by calibration problems (see Sect. 3.2).

B0742+103: neither Stickel et al. (1996) nor de Vries et al. (1995) were able to identify the host of this relatively bright radio source. Our UT1 image (Fig. 4), which unambiguously identifies the source with a compact host galaxy, indicates that the tentative identifications by Fugmann et al. (1988) and de Vries et al. (2000b) were correct: we establish $R=23.1$. We obtained long slit spectra of this source both in the January and the December run. Both observations show a faint spectrum and features which could be lines. However, none of these features are present in both spectra. Best et al. (2003) measure $2.624 \pm$ 0.003 based on Ly $\alpha$, C IV, He II and C III] between 4400 and $6920 \AA$ A. We detect a faint (slightly brighter than noise) emission at $6922 \AA$ which could be the Best et al. (2003) C III]. Having only one emission feature, we cannot get an independent redshift measurement. If their redshift is correct, using the $5 \mathrm{GHz}$ flux density from O'Dea (1998), the source would have a radio power of $2.1 \times 10^{28} \mathrm{~W} / \mathrm{Hz}$.

B0904+039: the deep UT1 image (Fig. 4) reinforces the earlier identification of this GPS radio source (de Vries et al. 2000b, $I=22.5)$ with a faint host $(V=24.9)$ in a group of faint galaxies. The spectrum shows a weak continuum (Fig. 3). We measure $z=$ 0.830 based on the [O II] and [O III] lines.

B0914+114: our $B$ and $V$ band images show an empty field at the accurate FIRST survey radio coordinates (Fig. 4). Based on uncertain WSRT coordinates, Stanghellini et al. (1993) suggested that the disk galaxy $\sim 6^{\prime \prime}$ south of the current radio position could be the host of the radio source. Thus, this disk galaxy is not the optical counterpart of the GPS source. We obtained a spectrum of the disk galaxy, which shows a faint stellar dominated continuum (Fig. 3). We observe narrow $\mathrm{H} \alpha$ and $\mathrm{H} \beta$ in emission on top of a broad absorption which also may be affecting [N II] and [S II] emission. Averaging the redshifts of all the observed features, we obtain $z=0.178$. The $\mathrm{H} \alpha$ emission at $7772 \AA$ had been detected before (de Vries et al. 2000b).

B1045+019: the weak continuum and noisy spectrum makes it difficult to distinguish real emission lines from noise. We only find one - dubious - possibility for redshift $(z=0.689)$. If this redshift is correct, we are not detecting $\mathrm{H} \beta$ and [O III] 5007 (at 8200 and $8550 \AA$ respectively). Radio observations were discussed in de Vries et al. (2000b) suggesting that this radio source may not be a GPS.

PMN J1300-1059: this source is from a list of candidate GPS sources found by comparing VLA NVSS and WSRT WISH survey data. We find one emission line at $6385 \AA$. The emission may consist of a narrow ( $~ 80 \AA)$ and a broad ( $200 \AA$ ) component but the edge of the chip is too close to deblend it accurately. This line could be $\operatorname{Mg}$ II $\lambda 2799$ at $z=1.283$, and in that case the [O II] doublet at $3727 \AA$ is not detected. If it were C IV $\lambda 1549$, we would expect C III] $\lambda 1909$ at $7826 \AA$. The relatively bright continuum suggests a QSO, which would be consistent with broad $\mathrm{Mg}$ II.

B1433-040: de Vries et al. (2000b) already drew attention to the fact that the GPS source B1433-040 should not be identified with the considerably brighter radio source $4 \mathrm{C}-04.51$. The optical spectrum shows a very strong continuum with broad

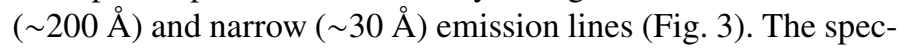
tral shape and presence of bright broad lines is consistent with a QSO. We observe a strong asymmetry in the broad emission. The asymmetry index (AI20, Heckman et al. 1981) is defined as (WL20-WR20)/(WL20+WR20), where WL20 and WR20 are the half width of the line to the left (WL20) and right (WR20) at the $20 \%$ intensity level. We measure AI20 0.35, towards the red, for $\mathrm{H} \beta$ (and $\mathrm{H} \gamma$ ), which is large, but not unusual. This asymmetry can be explained by inflow or outflow of gas, together with a line opacity (or scatter) cloud which blocks the 

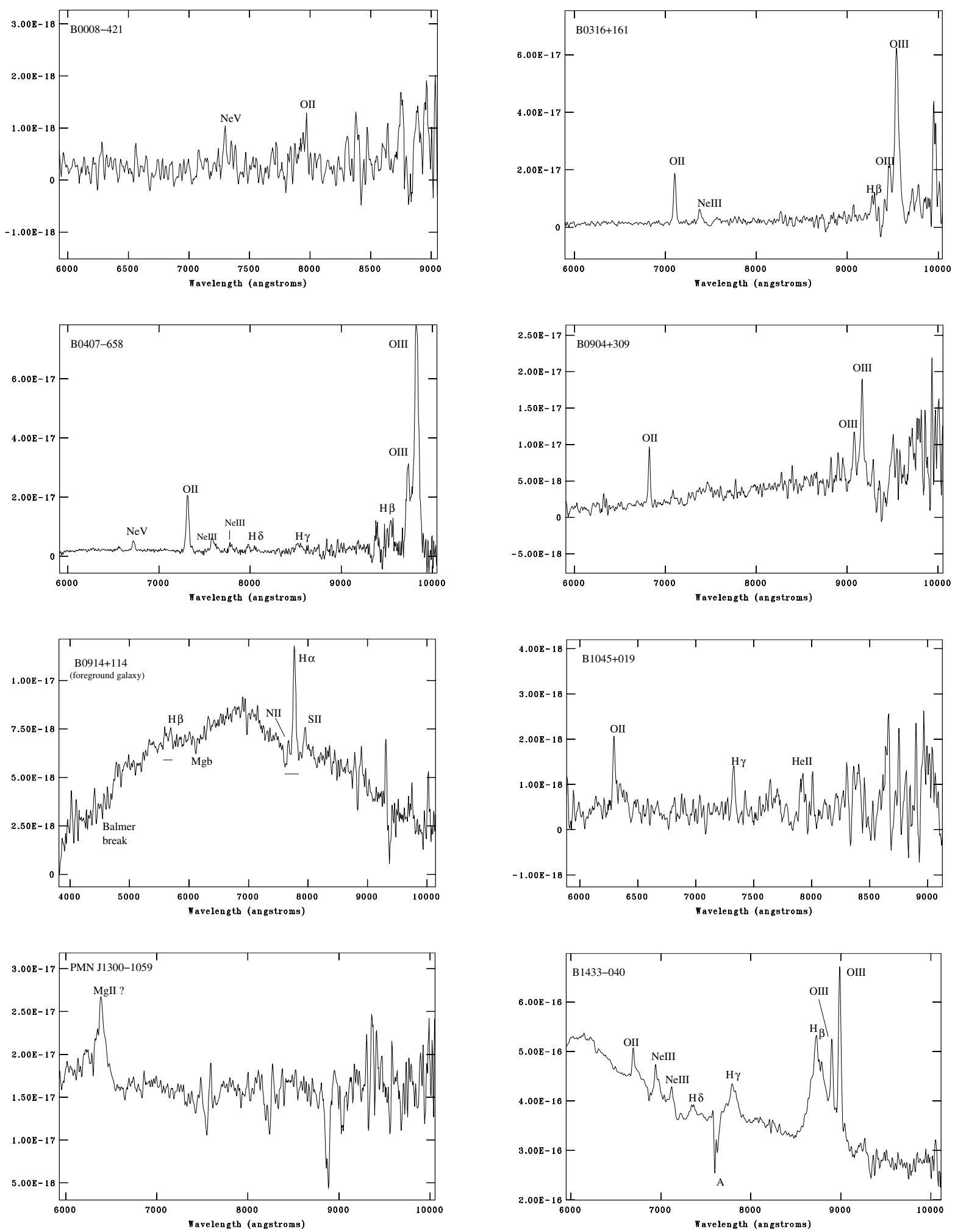

Fig. 3. Spectra of the sources with identified emission lines. Flux is in $\mathrm{erg} \mathrm{cm}^{-2} \mathrm{~s}^{-1} \AA^{-1}$. B0914+114: the spectrum corresponds to the previous incorrect - identification (see text for details). The horizontal lines show an estimation of the possible $F W H M$ of the absorption hydrogen lines.

emission at one side of the AGN (e.g., Whittle 1985, and references therein). The optical spectrum of this radio source displays hydrogen emission lines of striking velocity width: we measure $28000 \mathrm{~km} \mathrm{~s}^{-1}$ FWZI for $\mathrm{H} \beta$, and note in addition its doublepeaked nature. The radio spectrum has a relatively broad peak around $1 \mathrm{GHz}$ (e.g., Spoelstra et al. 1985). However, more observations, especially at frequencies $\lesssim 1 \mathrm{GHz}$, are needed. There seems to be some variability in the $408 \mathrm{MHz}$ and $\sim 1.4 \mathrm{GHz}$
(Large et al. 1981; Wright \& Otrupcek 1990; White \& Becker 1992) observed flux densities. Given the unresolved optical host, we suggest that the optical counterpart of B1433-040 is a quasar at $z=0.796$.

B1601-222: featureless, very noisy spectrum with a moderately bright continuum. Snellen et al. (2002) measure $z=$ 0.141 based on the absorption features: $G$-band $4300, \mathrm{H} \beta 4861$, Mg b 5169 and Na D 5899, which correspond to 4900-6800 ̊. 

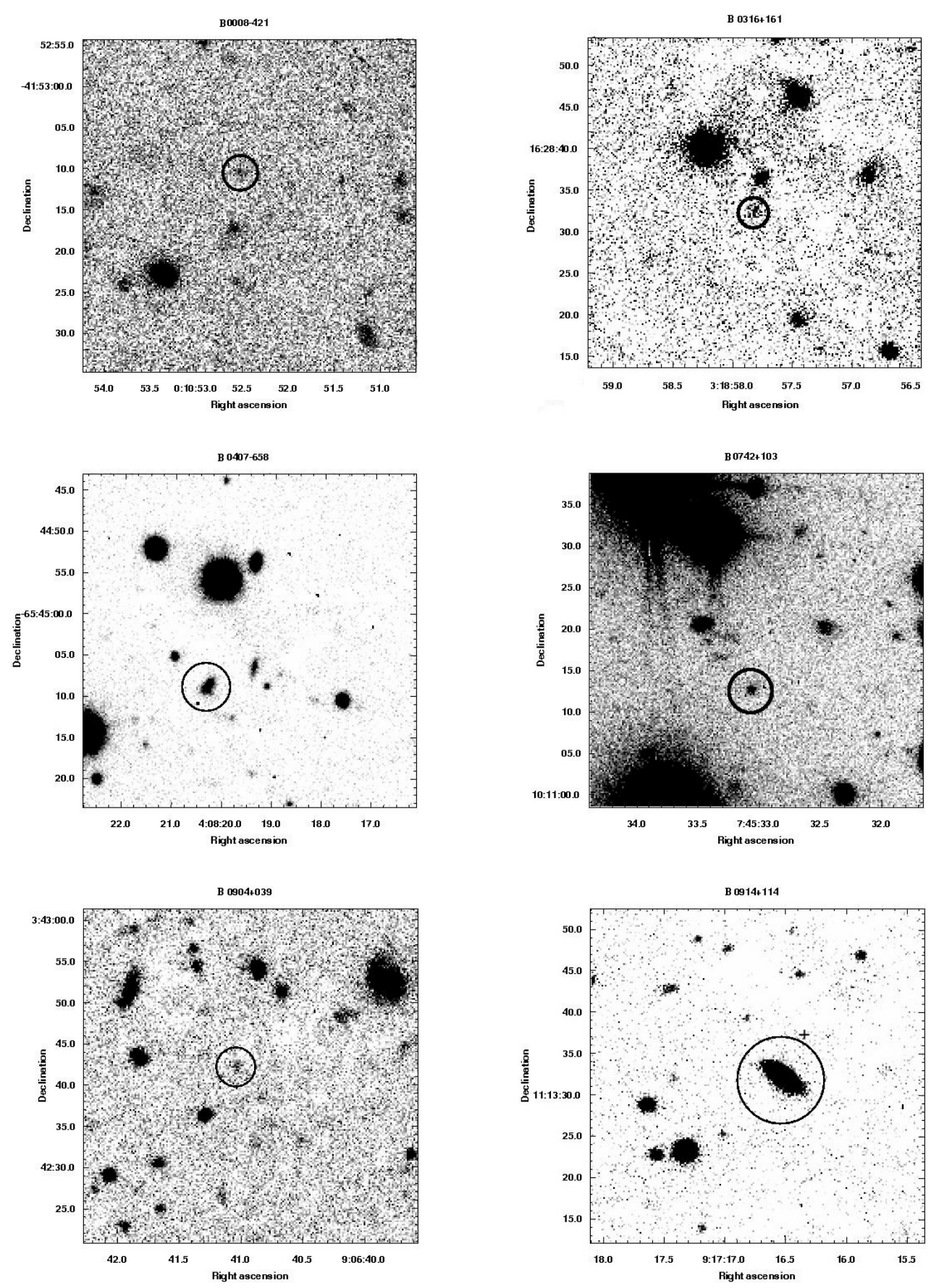

Fig. 4. Finding charts for the identified sources. The images correspond to the band of our VLT observations were sources were brighter. The radio position of $0914+114$ is $6^{\prime \prime}$ north of the observed galaxy (circled) which was previously - and incorrectly - identified as the optical counterpart (see text). The radio position is marked with a cross.

We covered the range between 5700 and $9200 \AA$ and observe no features. However, their spectrum has higher wavelength resolution and longer exposure time (2700 s).

B1648+015: featureless with a not very bright continuum. Stickel et al. (1996) identifies this source as a quasar. The radio spectrum shows variability so it is probably either a flat spectrum quasar or a BL Lac object.

\section{Master list}

We have updated the O'Dea et al. (1991) GPS sources master list with new available data (Table 4). This new list consists of those radio sources with intrinsic turnover frequency between
0.4 and $5 \mathrm{GHz}$ (GPS) or higher (High Frequency Peakers, HFP). For sources with unknown redshift, we have extended the selection down to $0.3 \mathrm{GHz}$. We expect this list to be useful for workers in the field of radio galaxies and quasars in general, and compact radio sources and AGN hosts in particular. The list is furthermore electronically available via URL http://www . damir.iem.csic.es/extragalactic/people/ labiano/ and http://www. cis.rit. edu/ cposps/.

\section{Summary}

We presented VLT deep optical imaging and spectroscopy targeting the host galaxies of GPS radio sources. The sample was 
comprised of unidentified objects from the master list of O'Dea et al. (1991), updated by de Vries et al. (1997).

We have found new optical counterparts (down to magnitudes 25 ) of GPS sources B0008-421 and B0742+103 and confirmed previous identifications of GPS sources: B0316+161, B0407-658, B0554-026, B0904+039. With new radio observations from the literature, we find that the radio spectra of B0407-658, B0437-454 and B1648+015 suggest that these sources are not GPS. However, high resolution radio observations are needed to confirm it. We do not detect the optical counterpart of B0914+114 and suggest that previous identification corresponds to an unrelated galaxy (at $z=0.178$ ), 6" South of the current radio position.

We measure new redshifts for B0316+161, B0407-658, B0904+039, B0914+114 (unrelated galaxy) and B1433-040, and propose uncertain redshifts for B0008-421, B1045+019, PMN J1300-1059. The following sources remain with undetermined redshift: B0437-454, B0914+114 and B1648+015. We cannot confirm previous redshifts of: B0742+103, B1601-222. Our magnitudes seem to be consistent with previous measurements of GPS counterparts.

Stellar population synthesis models are inconsistent with constant or exponentially declining star formation in the host. The data generally agree with single instantaneous burst models in a passively evolving host, but do not yield useful information on age or metallicity.

Acknowledgements. A.L. wishes to thank Prof. Dr. R. F. Peletier (Kapteyn Astronomical Institute) for useful scientific discussions. This paper is based on observations made with ESO Telescopes at Paranal Observatory under Programmes 64.P-0482(A) and 68.B-0044(A)). This research has made use of NASA's Astrophysics Data System Bibliographic Services and of the NASA/IPAC Extragalactic Database (NED) which is operated by the Jet Propulsion Laboratory, California Institute of Technology, under contract with the National Aeronautics and Space Administration. WDVs work was performed under the auspices of the US Department of Energy, National Nuclear Security Administration by the University of California, Lawrence Livermore National Laboratory under contract No. W-7405-Eng-48.

\section{References}

Appenzeller, I., Fricke, K., Furtig, W., et al. 1998, The Messenger, 94, 1 Augusto, P., Gonzalez-Serrano, J. I., Perez-Fournon, I., \& Wilkinson, P. N. 2006, MNRAS, 368, 1411

Beasley, A. J., Gordon, D., Peck, A. B., et al. 2002, ApJS, 141, 13

Becker, R. H., White, R. L., \& Edwards, A. L. 1991, ApJS, 75, 1

Begelman, M. C. 1996, in Cygnus A - Study of a Radio Galaxy, 209

Best, P. N., Peacock, J. A., Brookes, M. H., et al. 2003, MNRAS, 346, 1021

Bruzual, G., \& Charlot, S. 2003, MNRAS, 344, 1000

Chabrier, G. 2003, PASP, 115, 763

Condon, J. J., Cotton, W. D., Greisen, E. W., et al. 1998, AJ, 115, 1693

Costa, E. 2001, A\&A, 367, 719

Dallacasa, D., Stanghellini, C., Centonza, M., \& Fanti, R. 2000, A\&A, 363, 887

De Breuck, C., Tang, Y., de Bruyn, A. G., Röttgering, H., \& van Breugel, W. 2002, A\&A, 394, 59

de Ruiter, H. R., Arp, H. C., \& Willis, A. G. 1977, A\&AS, 28, 211

de Vries, W. H., Barthel, P. D., \& Hes, R. 1995, A\&AS, 114, 259

de Vries, W. H., Barthel, P. D., \& O’Dea, C. P. 1997, A\&A, 321, 105

de Vries, W. H., O'Dea, C. P., Baum, S. A., et al. 1998, ApJ, 503, 156

de Vries, W. H., O'Dea, C. P., Barthel, P. D., et al. 2000a, AJ, 120, 2300 de Vries, W. H., O’Dea, C. P., Barthel, P. D., \& Thompson, D. J. 2000b, A\&AS, 143,181

De Young, D. S. 1997, ApJ, 490, L55

di Serego-Alighieri, S., Danziger, I. J., Morganti, R., \& Tadhunter, C. N. 1994, MNRAS, 269, 998

Edwards, P. G., \& Tingay, S. J. 2004, A\&A, 424, 91

Fanti, C., Fanti, R., Dallacasa, D., et al. 1995, A\&A, 302, 317

Fugmann, W., Meisenheimer, K., \& Roeser, H.-J. 1988, A\&AS, 75, 173

Griffith, M. R., Wright, A. E., Burke, B. F., \& Ekers, R. D. 1994, ApJS, 90, 179

Heckman, T. M., Miley, G. K., van Breugel, W. J. M., \& Butcher, H. R. 1981, ApJ, 247, 403

Kaiser, C. R., \& Alexander, P. 1997, MNRAS, 286, 215

Kaiser, C. R., Dennett-Thorpe, J., \& Alexander, P. 1997, MNRAS, 292, 723

Kent, S. M. 1985, PASP, 97, 165

Kuehr, H., Witzel, A., Pauliny-Toth, I. I. K., \& Nauber, U. 1981, A\&AS, 45, 367

Labiano, A., O’Dea, C. P., Barthel, P. D., de Vries, W. H., \& Baum, S. A. 2006, New A Rev., 50, 776

Labiano, A., O’Dea, C. P., Barthel, P. D., de Vries, W. H., \& Baum, S. A. 2007, in prep.

Landolt, A. U. 1992, AJ, 104, 372

Large, M. I., Mills, B. Y., Little, A. G., Crawford, D. F., \& Sutton, J. M. 1981, MNRAS, 194, 693

Marcha, M. J. M., Browne, I. W. A., Impey, C. D., \& Smith, P. S. 1996, MNRAS, 281,425

McLean, B., Hawkins, C., Spagna, A., et al. 1998, in New Horizons from MultiWavelength Sky Surveys, ed. B. J. McLean, D. A. Golombek, J. J. E. Hayes, \& H. E. Payne, IAU Symp., 179, 431

Morganti, R., Killeen, N. E. B., \& Tadhunter, C. N. 1993, MNRAS, 263, 1023

Morganti, R., Tadhunter, C. N., Dickson, R., \& Shaw, M. 1997, A\&A, 326, 130 O'Dea, C. P. 1998, PASP, 110, 493

O'Dea, C. P., \& Baum, S. A. 1997, AJ, 113, 148

O'Dea, C. P., Baum, S. A., \& Stanghellini, C. 1991, ApJ, 380, 66

O’Dea, C. P., Stanghellini, C., Baum, S. A., \& Charlot, S. 1996, ApJ, 470, 806

Readhead, A. C. S., Taylor, G. B., Pearson, T. J., \& Wilkinson, P. N. 1996, ApJ, 460,634

Schlegel, D. J., Finkbeiner, D. P., \& Davis, M. 1998, ApJ, 500, 525

Sguera, V., Bassani, L., Malizia, A., et al. 2005, A\&A, 430, 107

Snellen, I. A. G., Bremer, M. N., Schilizzi, R. T., Miley, G. K., \& van Ojik, R. 1996, MNRAS, 279, 1294

Snellen, I. A. G., Schilizzi, R. T., de Bruyn, A. G., et al. 1998, A\&AS, 131, 435

Snellen, I. A. G., Schilizzi, R. T., Bremer, M. N., et al. 1999, MNRAS, 307, 149

Snellen, I. A. G., Schilizzi, R. T., Miley, G. K., et al. 2000, MNRAS, 319, 445

Snellen, I. A. G., Lehnert, M. D., Bremer, M. N., \& Schilizzi, R. T. 2002, MNRAS, 337, 981

Snellen, I. A. G., Mack, K.-H., Schilizzi, R. T., \& Tschager, W. 2004, MNRAS, 348,227

Spergel, D. N., Verde, L., Peiris, H. V., et al. 2003, ApJS, 148, 175

Spoelstra, T. A. T., Patnaik, A. R., \& Gopal-Krishna. 1985, A\&A, 152, 38

Stanghellini, C., O’Dea, C. P., Baum, S. A., \& Laurikainen, E. 1993, ApJS, 88, 1

Stickel, M., Rieke, G. H., Kuehr, H., \& Rieke, M. J. 1996, ApJ, 468, 556

Tadhunter, C. N., Morganti, R., di Serego-Alighieri, S., Fosbury, R. A. E., \& Danziger, I. J. 1993, MNRAS, 263, 999

Tinti, S., Dallacasa, D., de Zotti, G., Celotti, A., \& Stanghellini, C. 2005, A\&A, 432, 31

Torniainen, I., Tornikoski, M., Teräsranta, H., Aller, M. F., \& Aller, H. D. 2005, A\&A, 435, 839

Urry, C. M., \& Padovani, P. 1995, PASP, 107, 803

White, R. L., \& Becker, R. H. 1992, ApJS, 79, 331

Whittle, M. 1985, MNRAS, 213, 1

Wright, A., \& Otrupcek, R. 1990, in PKS Catalog (1990), 0

Wright, A. E., Griffith, M. R., Burke, B. F., \& Ekers, R. D. 1994, ApJS, 91, 111

Xiang, L., Reynolds, C., Strom, R. G., \& Dallacasa, D. 2006, A\&A, 454, 729

Xu, W., Lawrence, C. R., Readhead, A. C. S., \& Pearson, T. J. 1994, AJ, 108, 395

Xiang, L., Stanghellini, C., Dallacasa, D., \& Haiyan, Z. 2002, A\&A, 385, 768

Zensus, J. A., Ros, E., Kellermann, K. I., et al. 2002, AJ, 124, 662 
A. Labiano et al.: GPS radio sources: new optical observations and an updated master list, Online Material p 1

\section{Online Material}


A. Labiano et al.: GPS radio sources: new optical observations and an updated master list, Online Material p 2

Table 4. Master list.

\begin{tabular}{|c|c|c|c|c|c|c|c|c|c|c|c|}
\hline $\begin{array}{c}(1) \\
\text { Name }\end{array}$ & $\begin{array}{l}(2) \\
\text { Other }\end{array}$ & $\begin{array}{c}(3) \\
\text { GPS/HFP }\end{array}$ & $\begin{array}{l}4) \\
\text { ID }\end{array}$ & $\begin{array}{c}\text { (5) } \\
\text { Redshift }\end{array}$ & $\begin{array}{l}(6) \\
\text { Refs. }\end{array}$ & $\begin{array}{c}(7) \\
v_{\text {peak }} \\
(\mathrm{GHz})\end{array}$ & $\begin{array}{l}(8) \\
\text { Ref. }\end{array}$ & $\begin{array}{c}(9) \\
v_{\text {peakint }} \\
(\mathrm{GHz})\end{array}$ & $\begin{array}{l}(10) \\
\text { Flux } \\
\text { (Jy) }\end{array}$ & $\begin{array}{c}(11) \\
\text { Frequency } \\
(\mathrm{GHz})\end{array}$ & $\begin{array}{l}\text { (12) } \\
\text { Ref. }\end{array}$ \\
\hline $000319+212944$ & $0000+212$ & HFP & $\mathrm{G}$ & 0.4 & 5 & 6.2 & 6 & & 0.265 & 5 & 6 \\
\hline $000346+480703$ & $0001+478$ & GPS & & & 7 & 1 & $\mathrm{~N}$ & 1 & 0.197 & 4.85 & 9 \\
\hline $000520+052410$ & $0002+051$ & HFP & Q & 1.887 & 5 & 4.9 & 6 & 14.1 & 0.229 & 5 & 6 \\
\hline 001052-415310 & $0008-42$ & GPS & $\mathrm{G}$ & & 2 & 0.5 & $\mathrm{~N}$ & 0.5 & 1.12 & 5 & 10 \\
\hline $002127+731241$ & $0018+729$ & GPS & $\mathrm{G}$ & 0.821 & 8,29 & 1 & $\mathrm{~N}$ & 1.8 & 0.393 & 4.85 & 9 \\
\hline $002225+001456$ & $4 \mathrm{C}+00.02$ & GPS & G & 0.305 & 29 & 0.7 & 11 & 0.9 & 1.1 & 5 & 11 \\
\hline $002442-420203$ & $0022-423$ & GPS & Q & 0.937 & 2 & 1.6 & 0 & 3.1 & 1.7 & 5 & 0 \\
\hline $002914+345632$ & $0026+346$ & GPS & G & 0.517 & 1,12 & 1 & $\mathrm{~N}$ & 1.5 & 1.32 & 4.85 & 9 \\
\hline $003732+080813$ & $0034+078$ & HFP & $\mathrm{G}$ ? & $>1.8$ & 5 & 4.9 & 6 & $>13.7$ & 0.292 & 5 & 6 \\
\hline $004204+232001$ & $0039+230$ & GPS & & & 1 & $1 ?$ & $\mathrm{~N}$ & $1 ?$ & 1.65 & 4.85 & 9 \\
\hline 010813-120050 & 0105-122 & GPS & $\mathrm{G}$ & & 4 & 1 & 4 & 1 & 0.52 & 2.7 & 4 \\
\hline $011137+390628$ & $0108+388$ & HFP & G & 0.66847 & 1,29 & 4 & 11 & 6.7 & 1.26 & 5 & 11 \\
\hline $011638+242253$ & $0113+241$ & GPS & & & 5 & 4.9 & 6 & 4.9 & 0.243 & 5 & 6 \\
\hline $011935+321050$ & $4 C+31.04$ & GPS & G & 0.06 & 7,13 & 0.4 & $\mathrm{~N}$ & 0.4 & 1.59 & 4.85 & 9 \\
\hline $014658+211024$ & $0144+209$ & GPS & & & 1 & $1.3 ?$ & $\mathrm{~N}$ & $1.3 ?$ & 0.598 & 4.85 & 9 \\
\hline 015310-331025 & $0150-334$ & GPS & Q & 0.61 & 3 & 1.5 & 3 & 2.4 & 0.88 & 4.8 & 3 \\
\hline $020434+090349$ & $0201+088$ & GPS & & & 7 & $\sim 2$ & $\mathrm{~N}$ & $\sim 2$ & 0.774 & 4.85 & 9 \\
\hline $020346+113445$ & $0201+113$ & HFP & Q & 3.639 & 1,29 & $\sim 4$ & $\mathrm{~N}$ & 17.3 & 0.742 & 4.85 & 9 \\
\hline $020643-302458$ & 0204-306 & GPS & G & & 4 & 0.5 & 4 & 0.5 & 0.58 & 2.7 & 4 \\
\hline 021010-221336 & $0207-224$ & GPS & G & & 4 & 1.5 & 4 & 1.5 & 0.85 & 2.7 & 4 \\
\hline $021044+041934$ & $0208+040$ & GPS & & & 4 & 0.4 & 4 & 0.4 & 0.56 & 2.7 & 4 \\
\hline 024008-230915 & $0237-233$ & GPS & $\mathrm{Q}$ & 2.223 & 11,1 & 1 & 11 & 3.2 & 3.34 & 5 & 11 \\
\hline $024235-213226$ & $0240-217$ & GPS & G & 0.314 & 4 & 1 & 4 & 1.3 & 0.97 & 2.7 & 4 \\
\hline $025134+431515$ & $0248+430$ & HFP & Q & 1.32 & 1,29 & 7 & 29 & 16.2 & 1.24 & 5 & 30 \\
\hline $031857+162833$ & $4 C+16.09$ & GPS & Q & & 11 & 0.8 & 11 & 0.8 & 2.89 & 5 & 11 \\
\hline $032153+122113$ & $0319+121$ & GPS & Q & 2.662 & 1 & 0.4 & 11 & 1.5 & 1.1 & 5 & 11 \\
\hline $032320+053411$ & $4 C+05.14$ & GPS & G & 0.1785 & 4,14 & 0.4 & 4 & 0.5 & 1.6 & 2.7 & 4 \\
\hline $035721+231953$ & $0354+231$ & HFP & Q & & 5 & $>22$ & 6 & & 0.56 & 5 & 6 \\
\hline 040121-292126 & 0359-294 & GPS & G & & 4 & 0.4 & 4 & 0.4 & 0.58 & 2.7 & 4 \\
\hline $041046+765645$ & $4 C+76.03$ & GPS & G & 0.5985 & 1 & 0.6 & 0 & 1 & 2.82 & 5 & 0 \\
\hline 040757-275705 & $0405-280$ & GPS & G & & 4 & 1.5 & 4 & 1.5 & 0.93 & 2.7 & 4 \\
\hline 040734-392447 & 0405-395 & GPS & $\mathrm{G}$ & & 4 & 0.4 & 4 & 0.4 & 0.52 & 2.7 & 4 \\
\hline 042214-384452 & 0420-388 & GPS & Q & 3.11 & 1 & ? & $\mathrm{N}$ & ? & 0.13 & 4.85 & 26 \\
\hline $042746+413301$ & $0424+414$ & GPS & & & 7, & $\sim 2$ & $\mathrm{~N}$ & $\sim 2$ & 0.723 & 4.85 & 9 \\
\hline $043103+203734$ & $0428+205$ & GPS & G & 0.219 & 1,29 & 1.1 & 11 & 1.3 & 2.38 & 5 & 11 \\
\hline 043354-022956 & $4 C-02.17$ & GPS & G & & 4 & 0.4 & 4 & 0.4 & 1.04 & 2.7 & 4 \\
\hline 043701-184448 & 0434-188 & HFP & Q & 2.702 & 3 & 4.5 & 3 & 16.7 & 0.95 & 4.8 & 3 \\
\hline 044133-334003 & 0439-337 & GPS & & & 4 & 1.5 & 4 & 1.5 & 0.88 & 2.7 & 4 \\
\hline 045720-084905 & 0454-088 & GPS & G & & 4 & 0.4 & 4 & 0.4 & 0.58 & 2.7 & 4 \\
\hline $045952+022931$ & $0457+024$ & HFP & Q & 2.384 & 1,29 & 2.1 & 11 & 7.1 & 1.57 & 5 & 11 \\
\hline $050321+020305$ & $0500+019$ & GPS & Q & 0.58457 & 2,7 & 1.8 & 11 & 2.9 & 1.89 & 5 & 11 \\
\hline $051002+180042$ & $0507+179$ & GPS & Q & 0.3 & 29 & 1.4 & 0 & 1.8 & 0.73 & 5 & 0 \\
\hline 053008-250330 & $0528-250$ & HFP & Q & 2.813 & 1,29 & 2.7 & $\mathrm{~N}$ & 10.3 & 1.16 & 5 & 10 \\
\hline 055652-024105 & 0554-026 & GPS & G & 0.235 & 18 & 1 & 0 & 1.2 & 0.29 & 5 & 0 \\
\hline $062518+444002$ & $0621+446$ & HFP & & & 5 & 14 & 6 & & 0.442 & 5 & 6 \\
\hline $063802+593322$ & $0633+595$ & HFP & & & 5 & 12.9 & 6 & & 0.591 & 5 & 6 \\
\hline $064204+675836$ & $0636+680$ & HFP & Q & 3.18 & 1 & 3.7 & 6 & 15.5 & 0.474 & 5 & 6 \\
\hline 064425-345942 & 0642-349 & HFP & Q & 2.165 & 3 & 3.3 & 3 & 10.5 & 0.85 & 4.8 & 3 \\
\hline $064632+445117$ & $0642+449$ & HFP & Q & 3.396 & 5 & 15.5 & 6 & 68.1 & 1.896 & 5 & 6 \\
\hline $065031+600143$ & $0646+600$ & HFP & Q & 0.455 & 1,5 & 6.8 & 6 & 9.9 & 1.236 & 5 & 6 \\
\hline $070648+464756$ & $0703+468$ & GPS & Q? & & 7 & 0.5 & 0 & 0.5 & 0.62 & 4.85 & 9 \\
\hline $071338+434917$ & $0710+439$ & GPS & $\mathrm{G}$ & 0.518 & 1,11 & 1.9 & 11 & 2.9 & 1.68 & 5 & 11 \\
\hline
\end{tabular}


A. Labiano et al.: GPS radio sources: new optical observations and an updated master list, Online Material p 3

Table 4. continuned.

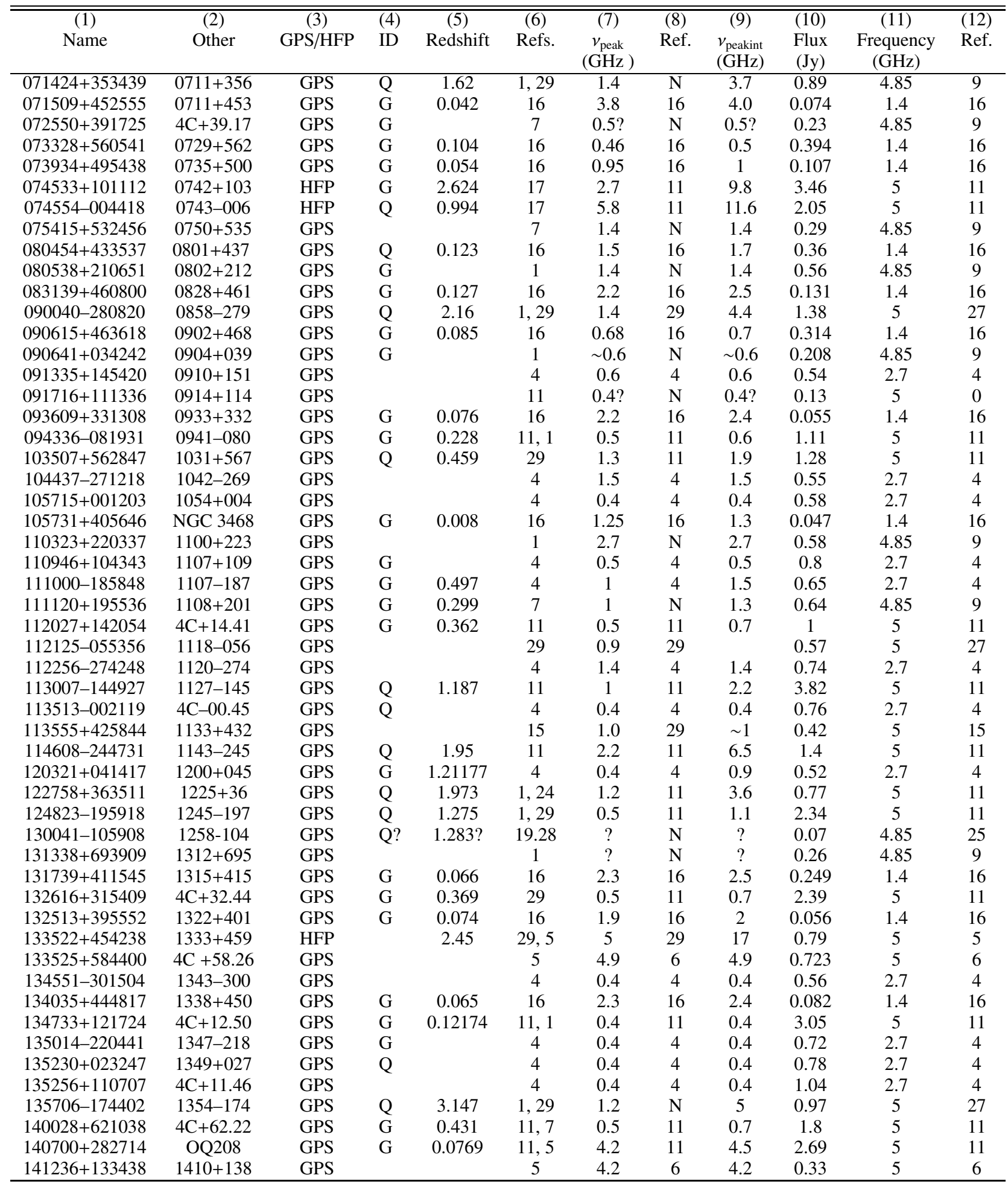


A. Labiano et al.: GPS radio sources: new optical observations and an updated master list, Online Material p 4

Table 4. continued.

\begin{tabular}{|c|c|c|c|c|c|c|c|c|c|c|c|}
\hline$\overline{~(1) ~}$ & $\overline{(2)}$ & $\overline{(3)}$ & (4) & 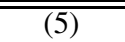 & $\overline{(6)}$ & $\overline{(7)}$ & $\overline{(8)}$ & $\overline{(9)}$ & $\overline{(10)}$ & $\overline{~(11) ~}$ & $\overline{(12)}$ \\
\hline Name & Other & GPS/HFP & ID & Redshift & Refs. & $\begin{array}{c}v_{\text {peak }} \\
(\mathrm{GHz})\end{array}$ & Ref. & $\begin{array}{l}v_{\text {peakint }} \\
(\mathrm{GHz})\end{array}$ & $\begin{array}{c}\text { Flux } \\
(\mathrm{Jy})\end{array}$ & $\begin{array}{c}\text { Frequency } \\
(\mathrm{GHz})\end{array}$ & Ref. \\
\hline $142438+225601$ & $1422+231$ & HFP & $\mathrm{Q}$ & 3.626 & 5 & 4.0 & 6 & 18.5 & 0.61 & 5 & 6 \\
\hline $143009+104328$ & $1427+109$ & HFP & $\mathrm{Q}$ & 1.71 & 5 & 4.9 & 6 & 13.3 & 0.91 & 5 & 6 \\
\hline 143539-041455 & $1433-04$ & GPS & $\mathrm{G}$ & 0.795 & 11 & 0.6 & $\mathrm{~N}$ & 1.1 & 0.2 & 5 & 0 \\
\hline $144516+095836$ & OQ172 & GPS & Q & 3.535 & 11 & 0.9 & 11 & 4.1 & 1.2 & 5 & 11 \\
\hline $144516+095836$ & 1444-339 & GPS & $\mathrm{G}$ & & 4 & 0.5 & 4 & 0.5 & 0.5 & 2.7 & 4 \\
\hline $150506+032630$ & $1502+036$ & HFP & Q & 0.411 & 5 & 6.2 & 6 & 8.8 & 0.93 & 5 & 6 \\
\hline 150603-091912 & $1503-091$ & GPS & $\mathrm{G}$ & & 4 & 0.6 & 4 & 0.6 & 0.87 & 2.7 & 4 \\
\hline $151141+051809$ & $1509+054$ & HFP & $\mathrm{G}$ & 0.084 & 5 & 11.0 & 6 & 11.9 & 0.54 & 5 & 6 \\
\hline $152114+043022$ & $4 C+04.51$ & GPS & Q & 1.296 & 29 & 0.8 & 11 & 1.8 & 1.09 & 5 & 11 \\
\hline $152237-273010$ & $1519-273$ & HFP & Q & 1.294 & 29,3 & 5.8 & 3 & 6.9 & 1.74 & 4.8 & 3 \\
\hline $152642+665054$ & $1526+670$ & HFP & $\mathrm{Q}$ & 3.02 & 5 & 5.8 & 6 & 23.3 & 0.41 & 5 & 6 \\
\hline $154301-075707$ & $1540-077$ & GPS & $\mathrm{G}$ & 0.172 & 4 & 0.4 & 4 & 0.5 & 1.21 & 2.7 & 4 \\
\hline $154609+002624$ & $1543+005$ & GPS & $\mathrm{G}$ & 0.556 & 18,4 & 1.2 & 0 & 1.9 & 0.84 & 5 & 0 \\
\hline $154812-121331$ & $1545-120$ & GPS & $\mathrm{G}$ & 0.883 & 4 & 0.4 & 4 & 0.8 & 1.45 & 2.7 & 4 \\
\hline $155614-062235$ & $4 C-06.43$ & GPS & $\mathrm{G}$ & & 4 & 0.4 & 4 & 0.4 & 0.77 & 2.7 & 4 \\
\hline $160000-003723$ & 1557-004 & GPS & & & 4 & 1 & 4 & 1 & 0.54 & 2.7 & 4 \\
\hline $160207+332653$ & $1600+335$ & GPS & $\mathrm{G}$ & 1.1 & 11,23 & 2.4 & 11 & 5 & 2.67 & 5 & 11 \\
\hline $160631+312710$ & $1604+315$ & GPS & G & $1.5 \mathrm{p}$ & 1,31 & & 1.5 & 29 & 0.08 & 4.8 & 9 \\
\hline $160913+264129$ & CTD93 & GPS & $\mathrm{G}$ & 0.473 & 1,29 & 1.1 & 11 & 1.6 & 1.73 & 5 & 11 \\
\hline $161637+045932$ & $1614+051$ & HFP & Q & 3.197 & 1,6 & 4.1 & 6 & 17.2 & 0.89 & 5 & 6 \\
\hline $162418-680913$ & $1619-680$ & GPS & Q & 1.36 & 3 & 3.1 & 3 & 7.3 & 1.69 & 4.8 & 3 \\
\hline $162304+662401$ & $1622+665$ & HFP & $\mathrm{G}$ & 0.203 & 5 & 5.1 & 6 & 6.1 & 0.3 & 5 & 6 \\
\hline $164047+122002$ & $4 C+12.6$ & GPS & $\mathrm{G}$ & 1.152 & 4 & 0.4 & 4 & 0.9 & 1.48 & 2.7 & 4 \\
\hline $164558+633011$ & $1645+635$ & HFP & Q & 2.379 & 5 & $>22$ & 6 & $>74.3$ & 0.51 & 5 & 6 \\
\hline $164831+024248$ & $4 C+02.43$ & GPS & & & 4 & 0.4 & 4 & 0.4 & 0.61 & 2.7 & 4 \\
\hline $165103+012923$ & $1648+015$ & GPS & Q & 0.4 & 0 & 1.5 & 0 & 2.1 & 1.03 & 5 & 0 \\
\hline 165844-073918 & $1656-075$ & GPS & & & 3 & 4.8 & 3 & 4.8 & 1.32 & 4.8 & 3 \\
\hline $172340-650036$ & $1718-649$ & GPS & G & 0.014 & 0 & 4 & 0 & 4.1 & 4.32 & 5 & 0 \\
\hline $172657-642753$ & $1722-644$ & GPS & & & 3 & 1.1 & 3 & 1.1 & 1.26 & 4.8 & 3 \\
\hline $173458+092657$ & $1732+094$ & GPS & $\mathrm{G}$ & $0.61 p$ & 7,4 & 2.8 & 0 & 4.5 & 0.86 & 5 & 0 \\
\hline $173549+504911$ & $1734+508$ & HFP & $\mathrm{G} ?$ & & 5 & 5.9 & 6 & & 0.97 & 5 & 6 \\
\hline $174425-514444$ & $1740-517$ & GPS & $\mathrm{G}$ & & 2 & $1 ?$ & $\mathrm{~N}$ & $1 ?$ & 3.9 & 5 & 27 \\
\hline $175301+275059$ & $1751+278$ & GPS & G & $0.86 p$ & 1,15 & $0.66 ?$ & $\mathrm{~N}$ & 1.2 & 0.27 & 5 & 15 \\
\hline $171854+544148$ & $1753+544$ & GPS & & 0.147 & 16 & 0.48 & 16 & 0.6 & 0.33 & 1.4 & 16 \\
\hline $180356+034108$ & $1801+036$ & GPS & $\mathrm{G}$ & & 7 & $?$ & $\mathrm{~N}$ & $?$ & 0.25 & 4.85 & 9 \\
\hline $181944+670847$ & $1819+671$ & GPS & $\mathrm{G}$ & 0.22 & 7,21 & $\sim 0.5$ & $\mathrm{~N}$ & 0.6 & 0.15 & 4.85 & 9 \\
\hline $182632+270808$ & $1824+271$ & GPS & $\mathrm{G} ?$ & & 15,29 & $1 ?$ & $\mathrm{~N}$ & $1 ?$ & 0.115 & 5 & 15 \\
\hline $183728-710844$ & $1831-711$ & HFP & Q & 1.356 & 3 & 8.2 & 3 & 19.3 & 2.39 & 4.8 & 3 \\
\hline $184057+390046$ & $1839+389$ & HFP & Q & 3.095 & 5 & 4.5 & 6 & 18.4 & 0.203 & 5 & 6 \\
\hline $184103+671849$ & $1841+673$ & GPS & $\mathrm{G}$ & 0.47 & 0 & $2 ?$ & $\mathrm{~N}$ & $2.9 ?$ & 0.16 & 5 & 9 \\
\hline $184535+354116$ & $1843+356$ & GPS & Q & 0.764 & 1,7 & 2 & 0 & 3.5 & 0.82 & 5 & 0 \\
\hline $185027+282514$ & $1848+283$ & HFP & $\mathrm{Q}$ & 2.56 & 1,5 & 8.3 & 6 & 29.5 & 1.246 & 5 & 6 \\
\hline $185527+374257$ & $1853+376$ & HFP & $\mathrm{G}$ & 0.5 & 5 & 4.5 & 6 & 6.8 & 0.36 & 5 & 6 \\
\hline 193925-634246 & 1934-638 & GPS & $\mathrm{G}$ & 0.183 & 2,1 & 1.4 & 0 & 1.7 & 6.5 & 5 & 0 \\
\hline $194553+705550$ & $1946+708$ & GPS & $\mathrm{G}$ & 0.101 & 7,21 & $\sim 0.6$ & $\mathrm{~N}$ & 0.7 & 0.64 & 4.85 & 9 \\
\hline 200324-325147 & $2000-330$ & HFP & Q & 3.773 & 1 & 5 & $\mathrm{~N}$ & 23.9 & 1.2 & 5 & 27 \\
\hline 201114-064403 & $2008-068$ & GPS & $\mathrm{G}$ & 0.547 & 4,29 & 1.4 & 11 & 2.2 & 1.34 & 5 & 11 \\
\hline $202135+051505$ & $2019+050$ & GPS & Q & & 5 & 3.7 & 6 & 3.7 & 0.477 & 5 & 6 \\
\hline $202456+171814$ & $2022+171$ & HFP & G & 0.9 & 5 & 14.5 & 6 & & 0.57 & 5 & 6 \\
\hline $205252+363535$ & $2050+364$ & GPS & $\mathrm{G}$ & 0.354 & 1,22 & 1.2 & $\mathrm{~N}$ & 1.6 & 3.4 & 4.85 & 9 \\
\hline $205828+054251$ & $4 C+05.78$ & GPS & $\mathrm{G}$ & 1.381 & 4 & 0.4 & 4 & 1 & 0.65 & 2.7 & 4 \\
\hline 212339-011234 & $2121-014$ & GPS & Q & 1.158 & 20 & 0.5 & 0 & 1.1 & 0.32 & 5 & 0 \\
\hline 212912-153841 & $2126-158$ & GPS & $\mathrm{Q}$ & 3.27 & 1,11 & 4.1 & 11 & 17.5 & 1.17 & 5 & 11 \\
\hline
\end{tabular}


A. Labiano et al.: GPS radio sources: new optical observations and an updated master list, Online Material p 5

Table 4. continuned.

\begin{tabular}{|c|c|c|c|c|c|c|c|c|c|c|c|}
\hline (1) & (2) & (3) & (4) & $\overline{(5)}$ & $\overline{(6)}$ & $\overline{(7)}$ & (8) & $\overline{(\overline{(9)}}$ & $\overline{c(10)}$ & $\overline{(11)}$ & 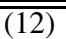 \\
\hline Name & Other & GPS/HFP & ID & Redshift & Refs. & $\begin{array}{c}v_{\text {peak }} \\
(\mathrm{GHz})\end{array}$ & Ref. & $\begin{array}{l}v_{\text {peakint }} \\
(\mathrm{GHz})\end{array}$ & $\begin{array}{l}\text { Flux } \\
(\mathrm{Jy})\end{array}$ & $\begin{array}{c}\text { Frequency } \\
(\mathrm{GHz})\end{array}$ & Ref. \\
\hline $213032+050217$ & $2128+048$ & GPS & $\mathrm{G}$ & 0.99 & 11,4 & 0.7 & 11 & 1.4 & 2.02 & 5 & 11 \\
\hline $215203-780707$ & $2146-783$ & GPS & Q & & 3 & 4.3 & 3 & 4.3 & 1.15 & 4.8 & 3 \\
\hline $215137+055213$ & $2149+056$ & GPS & $\mathrm{G}$ & 0.74 & 29 & 4.0 & 29 & 5.9 & 1.19 & 5 & 27 \\
\hline 215550-113948 & $2153-115$ & GPS & & & 1 & $1 ?$ & $\mathrm{~N}$ & $1 ?$ & 0.37 & 4.85 & 25 \\
\hline $221206+235540$ & $2209+236$ & HFP & Q & & 5 & 12.6 & 6 & & 1.18 & 5 & 6 \\
\hline $221237+015251$ & $4 C+01.69$ & GPS & G & & 11 & 0.5 & 11 & 0.5 & 1.05 & 5 & 11 \\
\hline $223834+124251$ & $2236+124$ & GPS & Q & & 1,29 & $5 ?$ & $\mathrm{~N}$ & $5 ?$ & 0.33 & 5 & 27 \\
\hline $225717+024317$ & $2254+024$ & HFP & Q & 2.081 & 5 & 19.5 & 6 & 60.1 & 0.274 & 5 & 6 \\
\hline $232510-034446$ & $2322-040$ & GPS & $\mathrm{G}$ & & 1,29 & 1.4 & 4 & 1.4 & 0.91 & 2.7 & 4 \\
\hline $232503+791716$ & $2323+790$ & GPS & G & & 1,29 & ? & $\mathrm{N}$ & ? & 1.136 & 1.4 & 19 \\
\hline $233013+334838$ & $2327+335$ & HFP & Q & 1.809 & 5 & 5.6 & 6 & 15.7 & 0.558 & 5 & 6 \\
\hline 233946-060412 & $4 C-06.76$ & GPS & G & & 4 & 0.4 & 4 & 0.4 & 0.8 & 2.7 & 4 \\
\hline $234029+264157$ & $2337+264$ & GPS & Q & & 1,29 & & & & & & \\
\hline $234403+822640$ & $2342+821$ & GPS & $\mathrm{Q}$ & 0.735 & 11,1 & 0.5 & 11 & 0.9 & 1.28 & 5 & 11 \\
\hline
\end{tabular}

Columns: (1) J2000 name. (2) B1950 or catalogue name. (3) GPS/HFP classification according to intrinsic peak frequency. (4) Optical identification: $\mathrm{G}=$ galaxy, $\mathrm{Q}=$ quasi-stellar object. (5) Redshift ( $\mathrm{p}=$ photometric). (6) Reference where the source is listed as a GPS, and references for optical ID and redshift. (7 and 8) Observed frequency of the spectral peak and reference. Data from NED were used to estimate the spectral peak for those sources with no published measurements of the peak frequency. "?" means that confirmation of the radio spectral shape seems necessary. (9) Intrinsic frequency of the spectral peak. (10,11 and 12) Flux density, frequency at which the flux density was measured, and reference.

References: 0 = This work. 1 = O'Dea et al. (1991), 2 = 2 Jy Sample: Morganti et al. (1993); Tadhunter et al. (1993); di Serego-Alighieri et al. (1994); Morganti et al. (1997), $3=$ Edwards \& Tingay (2004), $4=$ Snellen et al. (2002), $5=$ Tinti et al. (2005), $6=$ Dallacasa et al. $(2000), 7=$ Augusto et al. (2006), 8 = Snellen et al. (1996), $9=$ Becker et al. (1991), $10=$ Kuehr et al. (1981), $11=$ O'Dea (1998), 12=Zensus et al. (2002), 13 = Marcha et al. (1996), $14=$ Best et al. (2003), $15=$ Xiang et al. (2006), $16=$ CORALZ sample: Snellen et al. (2004), $17=$ Torniainen et al. (2005), 18 = de Vries et al. (2000b), $19=$ Condon et al. (1998), $20=$ de Vries et al. (1995), 21 = Snellen et al. (1999), $22=$ Beasley et al. (2002), $23=$ Snellen et al. (2000), $24=\mathrm{Xu}$ et al. (1994), $25=$ Griffith et al. (1994), $26=$ Wright et al. (1994), $27=$ Wright \& Otrupcek (1990), $28=$ De Breuck et al. (2002), $29=$ de Vries et al. (1997), $30=$ Snellen et al. (1998), 31 = Xiang et al. (2002), N = NASA/IPAC Extragalactic Database (NED). 